\title{
APLICAÇÃO DA METAHEURÍSTICA BUSCA TABU AO PROBLEMA DE ALOCAÇẤO DE AULAS A SALAS EM UMA INSTITUIÇÃO UNIVERSITÁRIA
}

\section{APPLICATION OF THE TABU SEARCH METAHEURISTIC TO THE CLASSROOM ASSIGNMENT PROBLEM IN A UNIVERSITY INSTITUTE}

\author{
Anand Subramanian* anand@ic.uff.br \\ José Maurício Fernandes Medeiros** mauricio@prr1.mpf.gov.br \\ Lucídio dos Anjos Formiga*** lucidio@di.ufpb.br \\ Marcone Jamilson Freitas Souza**** marcone@iceb.ufop.br \\ *Universidade Federal Fluminense (UFF) \\ ${ }^{* *}$ Ministério Público Federal (MPF)

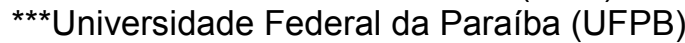 \\ ****Universidade Federal de Ouro Preto (UFOP)
}

\begin{abstract}
Resumo: Este artigo trata do Problema de Alocação de Aulas a Salas de uma Instituição Universitária. $\mathrm{Na}$ instituição analisada, a resolução deste problema é feita manualmente, tornando o processo árduo e demorado, além de frequentemente não produzir soluções que atendam a todas as restrições do problema. Desta forma, faz-se necessário automatizar o processo de alocação e, além disso, recorrer a estratégias computacionais que proporcionem soluções de qualidade e baixo custo. Devido à natureza combinatória do problema, recorreu-se à metaheurística Busca Tabu, que tem se mostrado adequada para a resolução desta classe de problemas. O algoritmo proposto parte de uma solução inicial gerada por um procedimento construtivo, o qual é capaz de produzir soluções viáveis em menos de um segundo. A seguir, esta solução é refinada pela Busca Tabu usando-se movimentos de realocação e troca de aulas entre salas para explorar o espaço de busca. $\mathrm{O}$ algoritmo proposto foi testado usando-se dados relativos à alocação de aulas de um semestre letivo e demonstrou ser bastante eficiente, tendo gerado soluções de alta qualidade quando comparado com a solução manual.
\end{abstract}

Palavras-chave: Problema de Alocação de Aulas a Salas, Otimização, Metaheurística, Busca Tabu, Instituição Universitária.

\begin{abstract}
This paper deals with the Classroom Assignment Problem in a University Institute. In the analyzed case, this problem is being resolved manually, which is an arduous and prolonged procedure, as well as often not capable of producing solutions that satisfy all the constraints. Thus there is a necessity to automatize the assignment process and to resort to computational strategies capable of yielding quality solutions at low costs. Due to the problem's combinatorial nature, use was made of the metaheuristic Tabu Search which has proven satisfactory for resolving these kinds of problems. The proposed method starts from an initial solution generated by a constructive procedure capable of producing feasible solutions in less than a second. Next, this solution is improved by the Tabu Search by applying movements that reallocate and exchange classes between classrooms in order to explore the solution space. The proposed algorithm was tested utilizing data related to the classroom assignment of a given semester and has demonstrated to be very effective, generating high quality solutions when compared to the manual solution.
\end{abstract}

Keywords: Classroom Assignment Problem, Optimization, Metaheuristic, Tabu Search, University Institution. 


\section{INTRODUÇÃO}

O Problema de Alocação de Aulas a Salas (PAAS) ou Classroom Assignment Problem consiste em alocar aulas, com horários de início e término previamente programados, a um número fixo de salas (CARTER \& LAPORTE, 1998; SCHAERF, 1999; SOUZA et al., 2002). Esse é um problema típico que surge nas instituições universitárias antes do início dos semestres letivos. Boa parte dessas instituições ainda resolve tal problema manualmente, o que torna o processo árduo e demorado, podendo levar vários dias para ser concluído. Ressalta-se ainda a sua importância quando se trabalha num contexto onde há escassez de salas de aula, que surge naturalmente com o crescimento do número de cursos e alunos da instituição.

O PAAS é um problema clássico de otimização combinatória pertencente à classe NP-hard (CARTER \& TOVEY, 1992), em que a determinação da solução ótima do problema, em um período de tempo aceitável, não é uma tarefa simples. Os métodos exatos, empregados na resolução de problemas que fazem parte desta classe, chegam a consumir tempos de ordem exponencial, ainda que sejam de dimensões medianas. Portanto, a utilização exclusiva de algoritmos exatos se torna praticamente inviável. Em vista disso, se faz necessário recorrer a outras técnicas na tentativa de se obter uma solução de qualidade, isto é, próxima à solução ótima e em tempos computacionais baixos. As técnicas heurísticas, de maneira geral, possuem esse perfil, sendo as metaheurísticas as mais indicadas por serem capazes de escapar de ótimos locais (SOUZA et al., 2002).

O presente trabalho propõe um algoritmo heurístico para resolver o PAAS em uma instituição universitária com o objetivo de se obter soluções com alto grau de satisfação e baixo custo computacional. $\mathrm{O}$ algoritmo desenvolvido é baseado na metaheurística Busca Tabu tendo em vista seu desempenho satisfatório na resolução de várias classes de problemas de programação de horários (WHITE et al., 2004; SANTOS et al., 2004; ALVAREZ-VALDÉS et al., 2001; HERTZ, 1991).

O restante do artigo está organizado da forma que se segue. A Seção 2 apresenta uma breve revisão bibliográfica sobre a Busca Tabu, destacando alguns trabalhos, relacionados ao PAAS, que fizeram uso desta metaheurística. A Seção 3 caracteriza o problema abordado neste trabalho. A Seção 4 traz os métodos e 
procedimentos utilizados. A Seção 5 discute os resultados computacionais obtidos. A Seção 6 apresenta as considerações finais deste artigo.

\section{REVISÃO BIBLIOGRÁFICA}

De acordo com Schaerf (1999), o PAAS é uma variante do problema básico de programação de horários de cursos universitários (course timetabling). Recentemente, problemas desta natureza vêm sendo tratados por meio de técnicas heurísticas, em particular, as metaheurísticas. Dentre as mais utilizadas, pode-se citar: Simulated Annealing, Algoritmos Genéticos, Colônia de Formigas e Busca Tabu, cujas descrições podem ser encontradas em Glover e Kochenberger (2004). Esta última metaheurística tem sido frequentemente empregada com êxito na resolução de problemas do gênero em questão. Hertz (1991), Carter e Tovey (1992), Costa (1994), Colorni et al. (1998), Schaerf (1999), Alvarez-Valdés et al. (2001), Souza et al. (2002), Willemen (2002), Santos et al. (2004), White et al. (2004) e Azimi (2005) obtiveram resultados significativos na resolução de problemas relacionados à programação de horários de instituições de ensino, bem como suas variantes, através da implementação deste método.

\subsection{Busca Tabu}

A metaheurística Busca Tabu (BT) foi inicialmente desenvolvida por Glover (1986) como uma proposta de solução para problemas de programação inteira. A partir de então, o autor formalizou esta técnica e publicou uma série de trabalhos contendo diversas aplicações da mesma. A experiência tem mostrado a eficiência da Busca Tabu na resolução de vários problemas de diferentes naturezas (GLOVER \& LAGUNA, 1993) e atualmente pode-se afirmar que se trata de uma técnica definitivamente consolidada.

Em linhas gerais, a Busca Tabu é um procedimento adaptativo, de busca local, dotado de uma estrutura de memória, que aceita movimentos de piora (quando não há possibilidades de melhora) para escapar de ótimos locais (HIGGINS, 2001; SOUZA, 2000). Sendo um procedimento de busca local, é baseado na noção de 
vizinhança. A cada iteração, a solução atual $s$ muda para outra que seja sua vizinha no espaço de busca, isto é, para uma solução $s$ ' que difere de $s$ por uma modificação. Partindo de uma solução inicial $s_{0}$, um algoritmo Busca Tabu explora, a cada iteração, um subconjunto $V$ da vizinhança $N(s)$ da solução corrente $s$. $O$ membro s' de $V$ com melhor valor nessa região segundo a função $f($.$) torna-se a$ nova solução corrente mesmo que $s^{\prime}$ seja pior que $s$ isto é, que $f\left(s^{\prime}\right)>f(s)$ para um problema de minimização.

O uso sistemático da memória é uma característica essencial da Busca Tabu. Enquanto a maioria dos processos de busca guarda essencialmente o valor de $f\left(s^{*}\right)$ da melhor solução $s^{*}$ obtida até o momento, a Busca Tabu arquiva as informações em uma espécie de itinerário das últimas soluções visitadas. Tais informações são utilizadas para conduzir o movimento de uma solução para outra a ser escolhida em $N(s)$. A função da memória consiste em restringir a escolha de algum subconjunto de $N(s)$, proibindo movimentos para algumas soluções vizinhas (HERTZ et al., 1995).

A proibição desses movimentos tem a intenção de impedir o retorno a uma solução já visitada anteriormente. O não veto de determinados movimentos pode fazer com que o algoritmo cicle. Um artifício criado com o intuito de não "autorizar" a ocorrência desses movimentos é a Lista Tabu $T$, que consiste em uma lista contendo as soluções visitadas durante as ultimas $|T|$ iterações sequenciadas na forma FIFO (First In First Out). Desta forma, o primeiro elemento a entrar na Lista Tabu é o primeiro a sair após $|T|$ iterações.

Os principais parâmetros de controle do método são o tamanho da Lista Tabu, a cardinalidade do subconjunto $V$ das soluções vizinhas testadas a cada iteração e o número máximo de iterações sem melhora na função objetivo (BTmax).

\section{CARACTERIZAÇÃO DO PROBLEMA}

O local selecionado para realização do estudo de caso foi o Centro de Tecnologia (CT) de uma Instituição de Ensino Superior. Este CT possui um total de 28 salas de aula sendo estas divididas em três tipos - com Carteira (C), com Mesa $(M)$ e com Prancheta $(P)$. As salas encontram-se distribuídas em seis diferentes blocos: A, B, D, E, F e H. A Tabela 1 ilustra as características de cada sala. 
Neste trabalho consideram-se apenas as turmas cujas aulas devem ser alocadas nas salas de aula mencionadas. As aulas ministradas em laboratórios não foram incluídas neste estudo. As aulas são ministradas nos turnos da manhã, tarde e noite, sendo que a maior demanda se dá no período matutino, em que o nível de ocupação das salas chega a $80,3 \%$ (vide Tabela 2), caracterizando a complexidade do PAAS neste período. Na Tabela 3, que mostra o percentual de ocupação das salas em cada turno e dia da semana, verifica-se que as terças e quartas pela manhã são os dias em que ocorrem as maiores demandas.

Todas as aulas ministradas no CT são destinadas às turmas dos seguintes cursos: Engenharia Mecânica (EM), Engenharia de Alimentos (EA), Química Industrial (QI), Engenharia Civil (EC), Engenharia de Produção Mecânica (EP) e Arquitetura e Urbanismo (AU). Com exceção deste último, os demais cursos possuem certas disciplinas cujas aulas são lecionadas em outros centros, e a responsabilidade de alocação das mesmas não cabe ao CT. Eventualmente, são alocadas turmas de outros centros nas salas do CT, mas isto só ocorre depois de realizada a alocação de todas as turmas referentes aos cursos supracitados. 
Tabela 1 - Características das salas do CT

\begin{tabular}{cccc}
\hline BLOCO & SALA & CAPACIDADE & TIPO \\
\hline \multirow{3}{*}{ A } & CTA 101 & 63 & $\mathrm{C}$ \\
& CTA 103 & 49 & $\mathrm{C}$ \\
& CTA 105 & 42 & $\mathrm{C}$ \\
& CTA 107 & 42 & $\mathrm{C}$ \\
$\mathrm{B}$ & CTB 101 & 42 & $\mathrm{C}$ \\
& CTB 102 & 15 & $\mathrm{C}$ \\
& CTB 103 & 35 & $\mathrm{C}$ \\
& CTB 105 & 30 & $\mathrm{C}$ \\
$\mathrm{D}$ & CTB 107 & 30 & $\mathrm{C}$ \\
& CTD 101 & 42 & $\mathrm{C}$ \\
& CTD 103 & 42 & $\mathrm{C}$ \\
$\mathrm{E}$ & CTD 105 & 42 & $\mathrm{C}$ \\
& CTD 107 & 42 & $\mathrm{C}$ \\
$\mathrm{F}$ & CTE 101 & 30 & $\mathrm{P}$ \\
& CTE 103 & 28 & $\mathrm{P}$ \\
& CTE 104 & 35 & $\mathrm{C}$ \\
& CTE105 & 26 & $\mathrm{C}$ \\
& CTF 101 & 25 & $\mathrm{M}$ \\
& CTH 101 & 16 & $\mathrm{C}$ \\
$\mathrm{H}$ & CTH 102 & 35 & $\mathrm{C}$ \\
& CTH 103 & 16 & $\mathrm{M}$ \\
& CTH 105 & 30 & $\mathrm{M}$ \\
& CTH AT1 & 25 & $\mathrm{M}$ \\
& CTH AT2 & 25 & $\mathrm{M}$ \\
& CTH AT3 & 25 & $\mathrm{M}$ \\
\hline
\end{tabular}

Tabela 2 - Ocupação das salas por turno

\begin{tabular}{cccc}
\hline TURNO & DISPONIBILIDADE $^{(*)}$ & DEMANDA $^{(*)}$ & PORCENTAGEM $^{(* 0}$ \\
\hline Manhã & 700 & 562 & $80,3 \%$ \\
Tarde & 700 & 391 & $55,9 \%$ \\
Noite & 700 & 68 & $9,7 \%$ \\
\hline
\end{tabular}

$\left({ }^{\star}\right)$ Em horas-aula por semana

Tabela 3 - Ocupação das salas por dia e turno

\begin{tabular}{cccccccc}
\hline DIA & $\begin{array}{c}\text { DISPONIBILIDADE } \\
\text { POR TURNO* }\end{array}$ & MANHÃ & $\%$ & TARDE & $\%$ & NOITE & $\%$ \\
\hline Segunda & 140 & 106 & 75,7 & 65 & 46,4 & 18 & 12,9 \\
Terça & 140 & 116 & 82,9 & 100 & 71,4 & 15 & 10,7 \\
Quarta & 140 & 119 & 85,0 & 73 & 52,1 & 17 & 12,1 \\
Quinta & 140 & 109 & 77,9 & 103 & 73,6 & 17 & 12,1 \\
Sexta & 140 & 112 & 80,0 & 50 & 35,7 & 1 & 0,71 \\
Total & 700 & 562 & & 391 & & 68 & \\
\hline
\end{tabular}

$\left(^{*}\right)$ Em horas-aula

A demanda semanal total corresponde a 1021 horas-aula. De acordo com o tipo de sala, a distribuição desta se dá conforme a Tabela 4. 
Tabela 4 - Demanda de acordo com o tipo de sala

\begin{tabular}{ccc}
\hline TIPO & DEMANDA $^{(*)}$ & $\%$ \\
\hline Carteira & 752 & 73,7 \\
Mesa & 235 & 23,0 \\
Prancheta & 34 & 3,33
\end{tabular}

$\left(^{*}\right)$ Em horas-aula por semana

A Tabela 5 mostra o número de turmas existentes no CT no primeiro semestre letivo de 2005 e suas respectivas demandas médias de alunos, separadas por curso(s). Algumas disciplinas possuem mais de uma turma, assim como existem turmas mistas, isto é, contendo alunos de cursos diferentes. Por exemplo, no referido semestre há 3 turmas, com demanda média de 20 alunos, contendo simultaneamente estudantes dos cursos EC e EA. A cada semestre letivo o número de turmas sofre uma ligeira alteração, porém, não há variação significativa.

Tabela 5 - Número de turmas e demanda média por Curso(s)

\begin{tabular}{ccc}
\hline CURSO(S) & NÚMERO DE TURMAS & $\begin{array}{c}\text { DEMANDA MÉDIA DE } \\
\text { ALUNOS }\end{array}$ \\
\hline EM & 29 & 15,82 \\
EA & 35 & 12,28 \\
QI & 12 & 9,69 \\
EC & 43 & 20,55 \\
EP & 44 & 12,66 \\
AU & 63 & 16,92 \\
EC e EM & 2 & 23,5 \\
EC, EA & 3 & 20 \\
EC, EM e EP & 2 & 34,5 \\
EC, EM, QI & 1 & 14 \\
EM e EP & 6 & 37 \\
EM e EA & 2 & 30,5 \\
EM, EA e QI & 2 & 27,5 \\
EA, QI & 2 & 10 \\
EC, EM, EA, QI & 3 & 40 \\
& Total de turmas $=248$ &
\end{tabular}

\subsection{Restrições do Problema}

As restrições do problema são as seguintes:

(R1) Duas ou mais aulas não podem ocorrer simultaneamente na mesma sala;

(R2) Aulas de uma determinada turma não podem ser alocadas em mais de uma sala num mesmo horário;

(R3) Aulas que necessitam de salas com mesa só podem ser alocadas em salas de seu respectivo tipo; 
(R4) Aulas que necessitam de salas com prancheta não podem ser alocadas em salas do tipo carteira;

(R5) Aulas de uma determinada turma só devem ser alocadas em salas de capacidade maior ou igual à demanda de estudantes desta;

(R6) O bloco F deve comportar somente aulas destinadas ao curso EP.

\subsection{Requisitos de Qualidade da Solução}

Os requisitos de qualidade descritos a seguir devem ser atendidos sempre que possível e o seu não atendimento não implica em inviabilidade.

(Q1) As aulas das turmas de um determinado curso e das turmas mistas devem ser alocadas em um bloco pré-determinado, conforme ilustra a Tabela 6. A razão pela qual a alocação destas aulas deve seguir esta recomendação é justificada pela relação entre as características físicas das salas de tal bloco e o perfil das disciplinas do(s) respectivo(s) curso(s). Por exemplo, o bloco H possui, em sua maioria, salas do tipo mesa, pois visa a atender aos requisitos de grande parte das disciplinas do curso $\mathrm{AU}$, que demandam este tipo de sala. Além disso, o bloco $\mathrm{H}$ pertence originalmente ao departamento responsável pelo curso AU. Por outro lado, o bloco B comporta laboratórios designados aos cursos EA e QI. Por isso disciplinas de ambos os cursos devem ser alocadas preferencialmente nesse bloco. Os demais casos seguem, basicamente, a mesma linha de raciocínio, porém, sempre havendo diferentes particularidades em cada curso.

(Q2) As aulas das disciplinas cuja frequência corresponde a dois dias semanais não devem ser alocadas em blocos distintos.

(Q3) As aulas das disciplinas cuja frequência corresponde a três dias semanais não devem ser alocadas em blocos distintos.

(Q4) As aulas das turmas que necessitam de carteiras, exceto as disciplinas do curso AU, não devem ser alocadas nos ateliês, localizados nas salas AT1, AT2, AT3, AT4, AT5 e AT6 do Bloco H.

(Q5) Aulas a serem ministradas, preferencialmente em salas com carteiras, não devem ser alocadas em salas do tipo mesa. 
(Q6) Aulas a serem ministradas, preferencialmente em salas com carteiras, não devem ser alocadas em salas do tipo prancheta.

(Q7) Aulas a serem ministradas em salas com pranchetas não devem ser alocadas a salas com mesas.

(Q8) Todas as aulas devem ser alocadas.

Tabela 6 - Alocação preferencial de turmas a um bloco pré-determinado

\begin{tabular}{cc}
\hline CURSO(S) & BLOCO \\
\hline EM & A \\
EA & B \\
QI & B \\
EC & D \\
EP & F \\
AU & H \\
EC e EM & E \\
EC, EA & $\mathrm{D}$ \\
EC, EM e EP & $\mathrm{D}$ \\
EC, EM, QI & $\mathrm{D}$ \\
EM e EP & $\mathrm{A}$ \\
EM e EA & $\mathrm{A}$ \\
EM, EA e Q & $\mathrm{A}$ \\
EA, QI & $\mathrm{B}$ \\
EC, EM, EA, QI & $\mathrm{D}$ \\
\hline
\end{tabular}

Os requisitos de qualidade (Q4) a (Q7) estão intrinsecamente relacionados à questão ergonômica, especialmente no que tange à biomecânica ocupacional. Isto se deve ao fato de que se uma determinada disciplina tiver sua aula alocada em uma sala cuja mobília não seja adequada para o cumprimento de suas devidas atividades, o aluno poderá estar sujeito a uma postura corporal inadequada, acarretando um possível estado de fadiga muscular e, consequentemente, comprometendo seu rendimento acadêmico.

A não alocação de todas as aulas não caracteriza, neste caso, uma inviabilidade da solução, pois, nestas situações, as mesmas podem ser alocadas em outros centros ou até mesmo em outras salas de aula do próprio CT, como, por exemplo, naquelas destinadas aos cursos de pós-graduação. Assim sendo, apesar de o não atendimento ao requisito (Q8) representar, a princípio, uma inviabilidade, ele foi tratado como sendo de qualidade. 


\subsection{Função de Avaliação}

Uma solução $s$ é avaliada por uma função $f$, dada pela expressão (1), a qual deve ser minimizada. A função de avaliação possui duas parcelas, sendo a primeira referente às penalizações pelo não atendimento às restrições (vide seção 3.1) e a segunda correspondente às penalizações pelo não atendimento aos requisitos de qualidade (vide seção 3.2):

$$
f(s)=R(s)+Q(s)
$$

sendo:

$$
R(s)=M \times \sum_{i=R 1}^{R 6} r_{i}(s)
$$

e

$$
Q(s)=\sum_{j=Q 1}^{Q 8} \alpha_{j} q_{j}(s)
$$

em que $r_{i}(s)$ e $q_{i}(s)$ correspondem, respectivamente, ao número de vezes (neste caso, a quantidade de horas-aula) que a restrição $i \in\{\mathrm{R} 1, \ldots, \mathrm{R} 6\}$ e o requisito de qualidade $j \in\{Q 1, \ldots, Q 8\}$ não é atendido na solução $s$. Nas expressões (2) e (3), $M$ é um parâmetro de penalidade associado ao não atendimento das restrições, enquanto $\alpha_{j} \geq 0$ é a penalidade associada ao requisito de qualidade $q_{j}$.

Para garantir que a solução do problema seja viável, $R(s)$ deve assumir valor zero, o que ocorre quando cada termo $r_{i}(s)$ se anula. Para alcançar este objetivo, o parâmetro de penalidade $M$ deve ter um valor suficientemente elevado na função de avaliação.

Os valores atribuídos às penalizações referentes aos requisitos de qualidade são diretamente proporcionais às suas respectivas relevâncias e devem ser parametrizados de acordo com o interesse do usuário. A ideia é que o mesmo possa gerar diferentes soluções, por meio da variação de tais penalidades e, então, efetuar sua escolha da solução. 


\section{MÉTODOS E PROCEDIMENTOS}

Os métodos e procedimentos utilizados para a resolução do PAAS estão subdivididos em dois momentos. O primeiro corresponde à geração de uma solução inicial de relativa qualidade e o segundo está relacionado à aplicação da metaheurística Busca Tabu, com o objetivo de aprimorar a solução inicialmente estabelecida.

\subsection{Geração da Solução Inicial}

A solução inicial é gerada por um procedimento construtivo determinístico. Seu pseudocódigo encontra-se exposto no Quadro 1.

O procedimento funciona da seguinte forma. Primeiramente carregam-se os dados referentes às salas e as turmas. Em seguida, as salas são designadas aos seus respectivos blocos e as turmas aos seus respectivos cursos, onde estas são dispostas em ordem decrescente de demanda.

A partir de então, as turmas são alocadas da seguinte maneira. Inicialmente, as turmas são alocadas às salas contidas nos blocos previamente determinados, conforme ilustra a Tabela 6, e com aulas a serem ministradas em uma mesma sala. Aquelas que não conseguirem ser designadas, são alocadas em diferentes blocos e não necessariamente na mesma sala. Se, porventura, a designação de uma determinada aula a uma sala, não tenha sido possível, esta é, então, alocada a uma sala virtual. Na verdade, trata-se de um artifício lógico criado para a situação em que uma turma não tenha conseguido ser alocada. 


\section{Procedimento GeracaoSolucaolnicial}

1. CarregarDados( );

2. para (bloco $=0$ até bloco $<$ total de blocos cadastrados) faça

3. para (curso $=0$ até curso $<$ total de cursos cadastrados) faça

4. Turmas[curso] $=$ PrepararTurmas(curso); $\quad$ \{Retorna uma lista das turmas de um dado curso em ordem decrescente de demanda\}

5. AlocarTurmas(Salas, Turmas) \{Tenta alocar as aulas das turmas de cada curso em seu respectivo bloco e em salas iguais $\}$

6. para todas as turmas de um determinado curso faça

7. para todas as salas de um determinado bloco faça

$8 . \quad$ se a sala tiver horários disponíveis então

9. Alocar( );

10. AlocarBlocosDistintos(Salas, Turmas)

$\{$ Tenta alocar as aulas das turmas ainda não alocadas em blocos distintos\}

11. para todas as turmas de um determinado curso faça

12. Se (turma não foi alocada) então

13. para todos os blocos faça

14. para todas as salas deste bloco faça

15. $\quad$ se a sala tiver horários disponíveis então

16. Alocar( );

$17 . \quad$ senão

18. $\quad$ se o bloco atual é o bloco virtual então

19. Cadastrar Sala Virtual \{Atualizar 'Turmas'\}

$20 . \quad$ Alocar( );

21. Retorna Solucaolnicial;

Fim GeracaoSolucaolnicial

Quadro 1 - Pseudocódigo do procedimento de geração da solução inicial

\subsection{Caracterização da Vizinhança e Algoritmo Busca Tabu}

Para explorar o espaço de soluções são usados dois tipos de movimento, realocação e troca, para definir as vizinhanças $N^{R}$ e $N^{T}$, respectivamente. O primeiro movimento consiste em alterar a sala em que uma aula deve ser ministrada; enquanto o segundo consiste em trocar a alocação de duas aulas ministradas em salas distintas. No problema considerado uma solução $s^{\prime}$ é dita vizinha de $s$ se $s^{\prime} \in$ $N(s)=N^{R}(s) \cup N^{T}(s)$.

Na Figura 1 ilustra-se a realocação das aulas da turma $X$, da sala 101 para a sala 103. Em (a) mostra-se o quadro de horários antes do movimento e em (b), depois do movimento. 
Na Figura 2 mostra-se o movimento de troca envolvendo as aulas das turmas A e D, que após o movimento são ministradas nas salas 103 e 101, respectivamente.

\begin{tabular}{|c|c|c|}
\hline Segunda-feira & \multicolumn{2}{|c|}{ Sala } \\
\hline Horário & 101 & 103 \\
\hline $07: 00-08: 00$ & $\mathbf{X}$ & \\
\hline $08: 00-09: 00$ & $\mathbf{X}$ & \\
\hline $09: 00-10: 00$ & & \\
\hline $10: 00-11: 00$ & & B \\
\hline 11:00-12:00 & & B \\
\hline
\end{tabular}

(a)

\begin{tabular}{|c|c|c|}
\hline Segunda-feira & \multicolumn{2}{|c|}{ Sala } \\
\hline Horário & 101 & 103 \\
\hline $07: 00-08: 00$ & & $\mathbf{X}$ \\
\hline $08: 00-09: 00$ & & $\mathbf{X}$ \\
\hline $09: 00-10: 00$ & & \\
\hline $10: 00-11: 00$ & & B \\
\hline $11: 00-12: 00$ & & B \\
\hline
\end{tabular}

(b)

Figura 1 - Movimento de realocação de uma aula

\begin{tabular}{|c|c|c|}
\hline Terça-feira & \multicolumn{2}{|c|}{ Sala } \\
\hline Horário & 101 & 103 \\
\hline 07:00-08:00 & & C \\
\hline 08:00-09:00 & & C \\
\hline 09:00-10:00 & A & D \\
\hline 10:00-11:00 & A & D \\
\hline 11:00-12:00 & A & \\
\hline
\end{tabular}

(a)

\begin{tabular}{|c|c|c|}
\hline Terça-feira & \multicolumn{2}{|c|}{ Sala } \\
\hline Horário & 101 & 103 \\
\hline $07: 00-08: 00$ & & C \\
\hline $08: 00-09: 00$ & & C \\
\hline $09: 00-10: 00$ & D & A \\
\hline $10: 00-11: 00$ & D & A \\
\hline 11:00-12:00 & & A \\
\hline
\end{tabular}

(b)

Figura 2 - Movimento de troca entre aulas que ocorrem em salas distintas

O Quadro 2 mostra como a metaheurística Busca Tabu foi adaptada para resolver o PAAS. Inicialmente é gerada uma solução inicial por meio da heurística exposta no Quadro 1. Em seguida, a cada iteração do método escolhe-se aleatoriamente uma aula. Para esta aula executa-se o melhor movimento com relação às vizinhanças de realocação e troca. O movimento não tabu dessa vizinhança restrita $V$ que produzir a solução com o valor mais favorável para a função de avaliação é realizado, independentemente de a solução gerada ser pior ou melhor que a solução corrente. Um movimento tabu também pode ser realizado, desde que produza uma solução melhor que a gerada até então (critério de aspiração por objetivo). Realizado um movimento, armazena-se na lista tabu o atributo < código da disciplina, bloco destino, sala de destino, bloco origem, sala origem> referente à aula que foi sorteada. A inserção desse atributo na lista impede que uma aula selecionada retorne à sua sala e bloco de origem por $|T|$ iterações. $O$ procedimento termina quando o número máximo de iterações (IterMax) for atingido 
ou quando o número máximo de iterações sem que haja melhora na função de avaliação (BTmax) for alcançado.

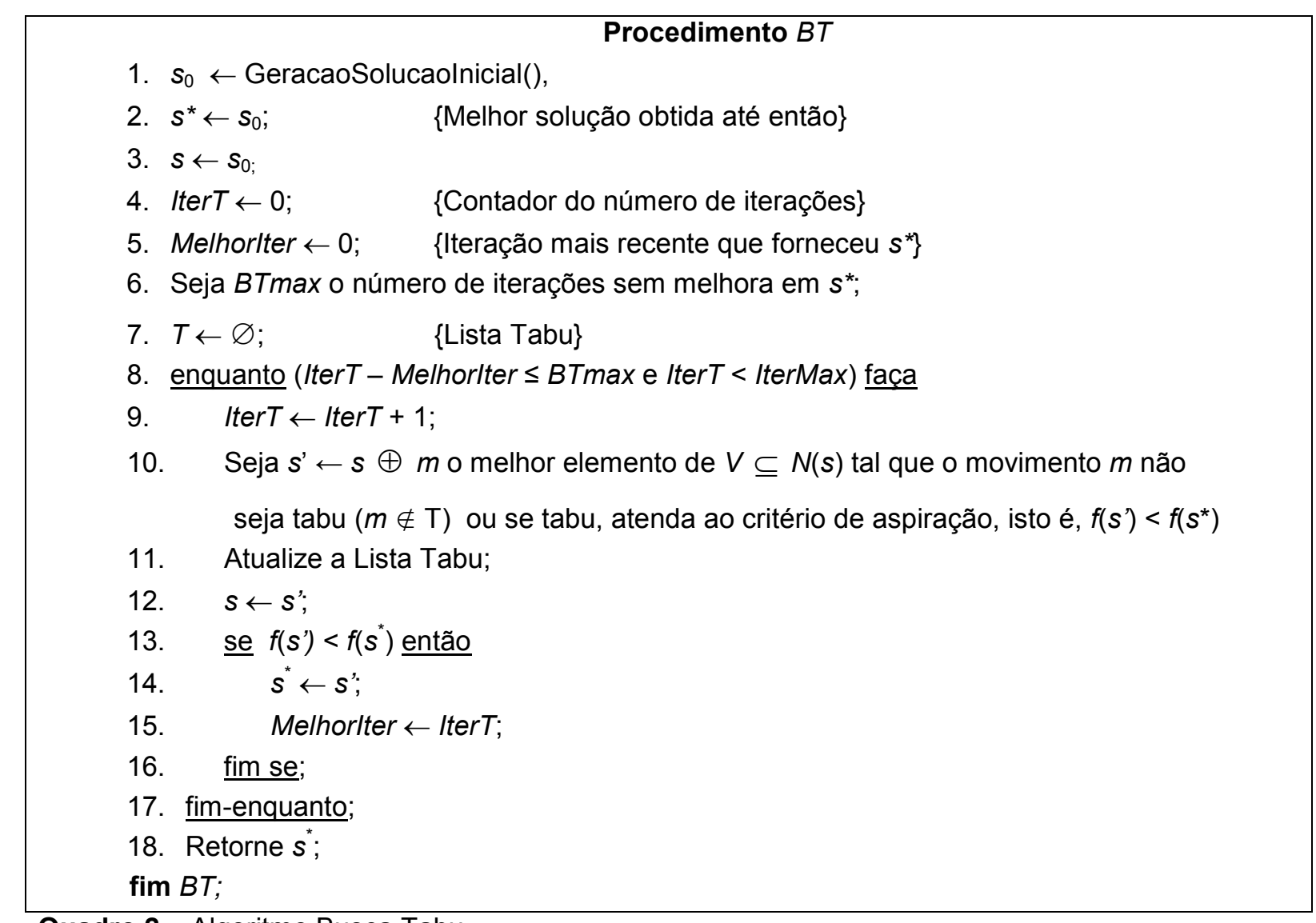

Quadro 2 - Algoritmo Busca Tabu

\section{RESULTADOS COMPUTACIONAIS}

O algoritmo proposto na seção 4 foi implementado na linguagem de programação $\mathrm{C}++$, utilizando o compilador Borland $\mathrm{C}++$ Builder 6.0 e executado em uma máquina com processador Intel Pentium Centrino 1,86 GHz com 1024 MB de memória RAM e sistema operacional Windows XP - Home Edition.

\subsection{Ajuste dos Parâmetros}


A calibração dos parâmetros do algoritmo proposto foi feita por meio de uma bateria de testes preliminares, sendo os valores adotados apresentados na Tabela 7.

Tabela 7 - Parâmetros do procedimento Busca Tabu

\begin{tabular}{cc}
\hline PARÂMETRO & VALOR \\
\hline IterMax (Número total de iterações) & 120000 \\
BTmax (Número de iterações sem & 20000 \\
melhora) & 100 \\
\hline$T \mid($ Tamanho da lista tabu) & \\
\hline
\end{tabular}

O algoritmo foi testado em dois cenários distintos com relação aos pesos conferidos aos requisitos de qualidade. A parametrização dos mesmos encontra-se na Tabela 8. O objetivo é avaliar a sensibilidade do algoritmo no que se diz respeito ao atendimento de tais requisitos em função da importância atribuída.

Tabela 8 - Pesos relativos aos requisitos de qualidade

\section{REQUISITO DE QUALIDADE}

(Q1) As aulas devem ser alocadas em blocos prédeterminados de acordo com a turma (Q2) As aulas das disciplinas cuja frequência corresponde a 2 dias semanais não devem ser alocadas em blocos distintos

(Q3) As aulas das disciplinas, cuja frequência corresponde a 3 dias semanais, não devem ser alocadas em blocos distintos

(Q4) Aulas a serem ministradas em salas do tipo carteiras não devem ser alocadas nos ateliês.

(Q5) Aulas a serem ministradas em salas do tipo carteiras não devem ser alocadas em salas com mesas

(Q6) Aulas a serem ministradas em salas do tipo carteiras não devem ser alocadas em salas com pranchetas

(Q7) Aulas a serem ministradas em salas do tipo prancheta não devem ser alocadas em salas com mesas

(Q8) Todas as aulas devem ser alocadas

\section{PESO}

$\alpha_{1}$ Cenário 2

Cenário 1 Cenário 2

$\begin{array}{lcc}\alpha_{2} & 1 & 100 \\ \alpha_{3} & 1000 & 100 \\ \alpha_{4} & 1000000 & 100 \\ \alpha_{5} & 1000 & 10 \\ \alpha_{6} & 100000 & 1 \\ & & 10 \\ \alpha_{7} & 1000000 & 100000000 \\ \alpha_{8} & 1000000000 & 100000000\end{array}$




\subsection{Comparação entre as Soluções Inicial, Final e Manual}

O algoritmo proposto foi executado 20 vezes em cada cenário, partindo de semente de números aleatórios diferentes.

A Tabela 9 detalha a quantidade, em horas-aula, de restrições violadas apenas na solução manual, uma vez que não houve inviabilidades nas soluções geradas pelo algoritmo proposto.

Tabela 9 - Inviabilidades da solução manual

\begin{tabular}{lcc}
\multicolumn{1}{c}{ RESTRIÇÃo } & $\begin{array}{c}\text { QUANTIDADE DE } \\
\text { OCORRÊNCIAS (EM } \\
\text { HORAS-AULA) }\end{array}$ & PERCENTUAL \\
\hline $\begin{array}{l}\text { R2) Aulas de uma determinada turma não podem } \\
\text { ser alocadas em mais de uma sala num mesmo } \\
\text { horário }\end{array}$ & 4 & $0,4 \%$ \\
$\begin{array}{l}\text { R3) aulas que necessitam de mesa só podem ser } \\
\text { alocadas em salas de seu respectivo tipo }\end{array}$ & 11 & $4,7 \%$ \\
$\begin{array}{l}\text { R5) Não se pode alocar aulas cujas turmas } \\
\text { apresentam uma demanda de alunos maior que a } \\
\text { capacidade da sala }\end{array}$ & 60 & $5,9 \%$ \\
$\begin{array}{l}\text { R6) Aulas de turmas não pertencentes ao curso de } \\
\text { EP não podem ser alocadas no Bloco F }\end{array}$ & 17 & $1,9 \%$ \\
\hline
\end{tabular}

A Tabela 10 mostra o relatório da execução dos procedimentos computacionais que deram origem às soluções inicial e final, bem como da solução obtida manualmente. Os resultados computacionais levaram em consideração as informações relacionadas ao não atendimento dos requisitos de qualidade, em horas-aula.

\begin{tabular}{c|cccc}
\multicolumn{5}{c}{ Tabela 10 - Comparação entre os resultados das soluções manual, inicial e final } \\
\hline $\begin{array}{c}\text { REQUISITO } \\
\text { DE }\end{array}$ & \multicolumn{4}{|c}{$\begin{array}{c}\text { QUANTIDADE DE HORAS-AULA EM QUE O REQUISITO NÃO FOI } \\
\text { QUTENDIDO }\end{array}$} \\
\cline { 2 - 5 } QUALIDADE & $\begin{array}{c}\text { Solução } \\
\text { manual }\end{array}$ & $\begin{array}{c}\text { Solução } \\
\text { inicial }\end{array}$ & $\begin{array}{c}\text { Cenário 1 } \\
\text { Solução final }\end{array}$ & Cenário 2 \\
\hline Q1 & 340 & 258 & $\mathbf{2 1 5}$ & 266 \\
Q2 & $\mathbf{2 3}$ & 97 & 130 & 33 \\
Q3 & $\mathbf{0}$ & 18 & 0 & 12 \\
Q4 & 35 & 23 & $\mathbf{0}$ & 2 \\
Q5 & 64 & $\mathbf{3 1}$ & 37 & 33 \\
Q6 & 42 & 50 & $\mathbf{2 7}$ & 45 \\
Q7 & 22 & $\mathbf{0}$ & $\mathbf{0}$ & $\mathbf{0}$ \\
Q8 & 48 & $\mathbf{0}$ & $\mathbf{0}$ & $\mathbf{0}$ \\
\hline
\end{tabular}


Com relação à solução manual, verifica-se, pela Tabela 9, que as restrições não foram atendidas em 92 horas-aula, o que representa 12,9\% do total a ser distribuído. Dentre estas, destaca-se o fato de que em 5,9\% do total de horas-aula, as turmas são alocadas a salas com capacidade inferior à demanda de alunos. Adicionalmente, pela Tabela 10, nota-se que 48 horas-aula sequer foram alocadas.

Por outro lado, apenas a fase construtiva do algoritmo proposto já foi suficiente para produzir uma solução inicial viável, além de ter sido capaz de alocar todas as aulas, conforme mostra a Tabela 10. Ressalta-se, ainda, que a solução inicial é obtida quase que instantaneamente, em menos de um segundo de processamento.

Tendo em vista a inviabilidade da solução manual, é feita a seguir apenas a comparação entre a solução final de cada cenário e a solução inicial.

No cenário 1, em foram dados maior importância aos requisitos de qualidade (Q8), (Q7), (Q4) e (Q6), observa-se que nos dois primeiros houve atendimento na íntegra, tal como na solução inicial, ao passo que os dois últimos foram substancialmente melhorados em $91,3 \%$ e 46,0\%, respectivamente. Nos requisitos de importância intermediária (Q1) e (Q3), houve melhoria de $16,7 \%$ no primeiro e pleno atendimento no segundo, ao contrário da solução inicial na qual havia 18 horas-aula não atendidas em relação a (Q3). Já o requisito (Q5) apresentou piora de $19,4 \%$. Entretanto, isto pode ser justificado pelo fato de (Q5) ter o mesmo peso de (Q1) e (Q3) e tratando-os em conjunto houve uma melhora de 17,9\%. O requisito de qualidade (Q2) apresentou uma substancial piora, de 34,0\%, porém isto pode ser facilmente justificado pelo fato deste apresentar relevância bem inferior às demais, ou seja, o atendimento a este requisito foi naturalmente "sacrificado" para que fossem privilegiados aqueles de maior expressividade.

No cenário 2 optou-se por utilizar uma configuração de pesos mais equilibrada em relação ao cenário 1 , à exceção do requisito (Q8), cujo atendimento integral foi mantido. Nos outros requisitos de maior importância, ou seja, (Q2), (Q3) e (Q4), houve melhora de $66,0 \%, 33,3 \%$ e $91,3 \%$, respectivamente, em relação à solução inicial. Nos requisitos de importância intermediária (Q5) e (Q7), houve piora de $6,5 \%$ no primeiro e manutenção do atendimento integral no segundo. No requisito (Q1) e (Q6) houve piora de $3,1 \%$ no primeiro e melhora de $10,0 \%$ no segundo. 
Contrariamente ao cenário 1, não houve piora substancial em nenhum dos requisitos, provavelmente devido ao fato de que os pesos adotados foram mais homogêneos.

\subsection{Variabilidade das Soluções Finais Geradas pelo Algoritmo}

Nesta seção, avalia-se o algoritmo com relação à variabilidade das soluções finais no que tange ao atendimento dos requisitos de qualidade.

As Tabelas 11 e 12 mostram os resultados das 20 execuções, bem como o tempo médio de execução do algoritmo proposto, nos cenários 1 e 2, respectivamente. Os números destacados em negrito correspondem à quantidade, em horas-aula, de violações aos requisitos de qualidade da melhor solução encontrada.

Tabela 11 - Resultados do cenário 1

\begin{tabular}{|c|c|c|c|c|c|c|c|c|c|}
\hline \multirow[t]{2}{*}{ Execução } & \multirow{2}{*}{$\begin{array}{c}\text { Tempo } \\
(\mathbf{s})\end{array}$} & \multicolumn{8}{|c|}{$\begin{array}{c}\text { Quantidade de horas-aula em que o requisito não foi } \\
\text { atendido }\end{array}$} \\
\hline & & (Q1) & (Q2) & (Q3) & (Q4) & (Q5) & (Q6) & (Q7) & (Q8) \\
\hline 1 & 1658 & 216 & 118 & 0 & 4 & 40 & 28 & 0 & 0 \\
\hline 2 & 1620 & 218 & 139 & 0 & 3 & 36 & 32 & 0 & 0 \\
\hline 3 & 1531 & 215 & 116 & 0 & 2 & 36 & 33 & 0 & 0 \\
\hline 4 & 1576 & 215 & 123 & 0 & 5 & 38 & 29 & 0 & 0 \\
\hline 5 & 1603 & 220 & 140 & 0 & 5 & 40 & 26 & 0 & 0 \\
\hline 6 & 1357 & 220 & 137 & 0 & 6 & 44 & 26 & 0 & 0 \\
\hline 7 & 1598 & 215 & 130 & 0 & 2 & 37 & 27 & 0 & 0 \\
\hline 8 & 1625 & 216 & 127 & 0 & 3 & 41 & 27 & 0 & 0 \\
\hline 9 & 1618 & 220 & 138 & 0 & 2 & 41 & 28 & 0 & 0 \\
\hline 10 & 1869 & 214 & 135 & 0 & 3 & 40 & 30 & 0 & 0 \\
\hline 11 & 1908 & 216 & 116 & 0 & 2 & 40 & 27 & 0 & 0 \\
\hline 12 & 1561 & 220 & 140 & 0 & 6 & 40 & 27 & 0 & 0 \\
\hline 13 & 1627 & 218 & 112 & 0 & 5 & 36 & 35 & 0 & 0 \\
\hline 14 & 1547 & 217 & 119 & 0 & 5 & 41 & 27 & 0 & 0 \\
\hline 15 & 2350 & 217 & 126 & 0 & 4 & 42 & 27 & 0 & 0 \\
\hline 16 & 1481 & 219 & 140 & 0 & 4 & 42 & 29 & 0 & 0 \\
\hline 17 & 1716 & 214 & 124 & 0 & 2 & 40 & 29 & 0 & 0 \\
\hline 18 & 1600 & 218 & 140 & 0 & 4 & 40 & 27 & 0 & 0 \\
\hline 19 & 1558 & 217 & 139 & 0 & 6 & 42 & 27 & 0 & 0 \\
\hline 20 & 1558 & 218 & 140 & 0 & 4 & 36 & 30 & 0 & 0 \\
\hline Média & 1648 & 217,2 & 130,0 & 0,0 & 3,9 & 39,6 & 28,6 & 0 & 0 \\
\hline $\begin{array}{l}\text { Desv. } \\
\text { Pad. }\end{array}$ & 203,8 & 2,0 & 10,0 & 0,0 & 1,4 & 2,3 & 2,4 & 0 & 0 \\
\hline
\end{tabular}

Pelas tabelas 11 e 12, observa-se que a carga horária média de violações aos requisitos de qualidade é bem próxima àquela referente à melhor solução. No cenário 1 , as violações médias diferem em no máximo 2,6 horas-aula da solução de 
valor mais favorável da função de avaliação, enquanto que no cenário 2 esta diferença é de no máximo 5,9 horas-aula. À exceção do requisito (Q2), o desviopadrão das violações foi baixo, sendo no máximo de 2,4 e 4,0 horas-aula nos cenários 1 e 2, respectivamente. Quanto ao desvio-padrão relativo ao requisito (Q2), é interessante notar que este tinha a menor relevância no cenário 1 e quando Ihe foi dada uma maior importância relativa (cenário 2), seu desvio-padrão diminuiu, mas à custa do aumento de todos os demais nos quais havia violação.

Tabela 12 - Resultados do cenário 2

\begin{tabular}{cccccccccc}
\hline & \multirow{2}{*}{$\begin{array}{c}\text { Tempo } \\
\text { Execução }\end{array}$} & \multicolumn{7}{c}{ Quantidade de horas-aula em que o requisito não foi } \\
\cline { 3 - 9 } & $\mathbf{( s )}$ & $\mathbf{( Q 1 )}$ & $\mathbf{( Q 2 )}$ & $\mathbf{( Q 3 )}$ & $\mathbf{( Q 4 )}$ & $\mathbf{( Q 5 )}$ & $\mathbf{( Q 6 )}$ & $\mathbf{( Q 7 )}$ & $\mathbf{( Q 8 )}$ \\
\hline 1 & 1550 & 264 & 32 & 18 & 4 & 33 & 46 & 0 & 0 \\
2 & 1209 & 264 & 28 & 24 & 6 & 32 & 51 & 0 & 0 \\
3 & 1648 & 258 & 43 & 18 & 4 & 34 & 47 & 0 & 0 \\
$\mathbf{4}$ & $\mathbf{1 5 9 9}$ & $\mathbf{2 6 6}$ & $\mathbf{3 3}$ & $\mathbf{1 2}$ & $\mathbf{2}$ & $\mathbf{3 3}$ & $\mathbf{4 5}$ & $\mathbf{0}$ & $\mathbf{0}$ \\
5 & 1582 & 262 & 49 & 6 & 2 & 31 & 46 & 0 & 0 \\
6 & 1293 & 262 & 38 & 18 & 2 & 33 & 43 & 0 & 0 \\
$\mathbf{7}$ & 1243 & 259 & 36 & 18 & 10 & 33 & 44 & 0 & 0 \\
8 & 1295 & 257 & 40 & 12 & 6 & 33 & 44 & 0 & 0 \\
9 & 1414 & 264 & 45 & 18 & 2 & 31 & 44 & 0 & 0 \\
10 & 1436 & 266 & 41 & 18 & 3 & 31 & 43 & 0 & 0 \\
11 & 1411 & 268 & 58 & 18 & 2 & 26 & 51 & 0 & 0 \\
12 & 1530 & 268 & 30 & 18 & 5 & 33 & 50 & 0 & 0 \\
13 & 1524 & 264 & 38 & 18 & 2 & 27 & 51 & 0 & 0 \\
14 & 1017 & 264 & 35 & 18 & 6 & 31 & 48 & 0 & 0 \\
15 & 1483 & 269 & 38 & 18 & 3 & 36 & 45 & 0 & 0 \\
16 & 1500 & 265 & 34 & 12 & 4 & 28 & 50 & 0 & 0 \\
17 & 1521 & 261 & 27 & 18 & 3 & 34 & 45 & 0 & 0 \\
18 & 1503 & 258 & 46 & 12 & 4 & 28 & 48 & 0 & 0 \\
19 & 1183 & 263 & 45 & 12 & 4 & 31 & 53 & 0 & 0 \\
20 & 1457 & 268 & 41 & 12 & 3 & 32 & 44 & 0 & 0 \\
Média & 1420 & 263,5 & 38,9 & 15,9 & 3,9 & 31,5 & 46,9 & 0 & 0 \\
Desv. & 163,1 & 3,6 & 7,6 & 4,0 & 2,0 & 2,5 & 3,1 & 0 & 0 \\
Pad. & & & & & & & & &
\end{tabular}

Os resultados completos das alocações feitas nos dois cenários estão disponíveis no endereço http://www.decom.ufop.br/prof/marcone/projects/paas.html.

\section{CONCLUSÕES}

Neste trabalho, realizou-se um levantamento da demanda correspondente à alocação das aulas no âmbito das salas disponíveis nos blocos do Centro de Tecnologia de uma Instituição Universitária. A partir disto, foi possível apresentar um 
diagnóstico preciso de ocupação das salas por turno, dia da semana e tipo de sala. Tal estudo permitiu aumentar a eficiência da gestão administrativa do Centro no que diz respeito à mudança de horários de determinada turma, ou até mesmo na definição de horários para novas turmas, exercendo-se assim um maior aproveitamento do conjunto de salas de aula. Foi constatada a existência de uma maior demanda no turno da manhã, onde cerca de $80 \%$ do total de horas-aula semanais encontram-se designadas neste período, tornando o processo de alocação de aulas a salas bastante complexo neste turno.

No tocante à otimização do processo, desenvolveu-se uma estratégia construtiva para gerar uma solução inicial, baseada no conhecimento prévio das características do problema. Esta abordagem construtiva foi capaz de gerar, quase que instantaneamente, soluções que contemplaram todas as restrições de viabilidade com um adicional qualitativo, se comparado com a solução obtida manualmente. Esta, por sua vez, não atendia plenamente aos critérios de viabilidade, bem como deixou de alocar 48 horas-aula (aproximadamente $4,7 \%$ do total).

A fase de refinamento do algoritmo proposto foi feita pela metaheurística Busca Tabu. Para testá-la, foram utilizados dois cenários que se diferiam com relação aos pesos atribuídos aos requisitos de qualidade. Em ambos, a Busca Tabu produziu soluções finais de qualidade compatível com os valores dos pesos considerados, mostrando-se adequada à resolução do Problema de Alocação de Aulas a Salas.

\section{AGRADECIMENTOS}

O último autor agradece à FAPEMIG, processo CEX PPM 00357/09, e ao CNPq, processo 482765/2010-0, pelo apoio recebido.

\section{REFERÊNCIAS}

ALVAREZ-VALDÉS, R.; CRESPO, E; TAMARIT, J. M. Tabu search: an efficient metaheuristic for university organization problems. Revista Investigacion

Operacional, v. 22, n. 2, p. 104-113, 2001. 
AZIMI, Z. N. Hybrid heuristics for examination timetabling problem. Applied Mathematics and Computation, v. 163, n. 2, p. 705-733, 2005.

BLUM, C.; ROLI, A. Metaheuristics in combinatorial optimization: overview and conceptual comparison. ACM Computer Surveys, v. 35, n. 3, p. 268-308, 2003.

CARTER, M. W.; LAPORTE, G. Recent developments in practical course timetabling. Lecture Notes in Computer Science, v. 1408, p. 3-19, 1998.

CARTER, M. W.; TOVEY, C.A. When is the classroom assignment problem hard?. Operations Research Supplement 1, v. 40, p. 28-39, 1992.

COLORNI, A.; DORIGO, M.; MANIEZZO, V.; Metaheuristics for high school timetabling. Computational Optimization and Applications, v. 9, n. 3, p. 275-298, 1998.

COSTA, D. A tabu search algorithm for computing an operational timetable. European Journal of Operational Research, v. 76, p. 98-110, 1994.

GLOVER, F. Future paths in Integer programming and links to artificial intelligence. Computers and Operations Research, v. 13, n.5, p. 533-549, 1986.

GLOVER, F.; KOCHENBERGER, G. A. Handbook of metaheuristics. Boston: Kluwer Academic Publishers, 2003.

GLOVER, F.; LAGUNA, M. Tabu search. Modern Heuristics Techniques for Combinatorial Problems, Blackwell Scientific Publications, Oxford, p. 70-150, 1993.

HERTZ, A. Tabu search for large scale timetabling problems. European Journal of Operational Research Society, v. 54, p. 39-47, 1991.

HERTZ, A.; TAILLARD, E.; de WERRA, D. A tutorial on tabu search. Proc. of Giornate di Lavoro AIRO'95, (Entreprise Systems: Management of Technological and Organizational Changes), p. 13-24, 1995.

HIGGINS, A. J. A dynamic tabu search for large-scale generalized assignment problems. Computers and Operations Research, v. 28, p. 1039-1048, 2001.

OSMAN, I. H. Focused issue on applied meta-heuristics. Computers and Industrial Engineering, v. 44, p. 205-207, 2002.

SANTOS, H .G.; OCHI, L. S.; SOUZA, M. J. F. An efficient tabu search heuristic for the school timetabling problem. Lecture Notes in Computer Science, v. 3059, p.468-481, 2004.

SCHAERF, A. A survey of automated timetabling. Artificial Intelligence Review, v. 13, p.87-127, 1999. 
SOUZA, M. J. F. Programação de horários em escolas: uma aproximação por metaheurísticas. Tese (Doutorado), Programa de Engenharia de Sistemas e Computação, COPPE/UFRJ, Rio de Janeiro, Brasil, 2000.

SOUZA, M. J. F., XAVIER, A. X.; ARAÚJO, C. R. Experiências com a utilização de Simulated Annealing e Busca Tabu na resolução do Problema de Alocação de Salas. In: SIMPÓSIO BRASILEIRO DE PESQUISA OPERACIONAL, 34, 2002, São Paulo. Anais... São Paulo: SBPO, p. 1100-1110, 2002.

WHITE, G. M.; Xie, B. S.; Zonjic, S. Using tabu search with longer-term memory and relaxation to create examination timetables. European Journal of Operational Research, v. 153, p. 90-91, 2004.

WILLEMEN, R. School timetable construction: algorithms and complexity. Tese (doutorado), Technische Universiteit Eindhoven, Holanda, 2002.

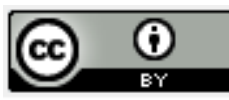

Artigo recebido em 09/08/2009 e aceito para publicação em 22/02/2011. 